\title{
A Quarter Century of Research Progress Leads to Answers and New Questions for Women's Sleep Health
}

\author{
Katherine M. Sharkey ${ }^{1}$ Amy R. Wolfson ${ }^{2}$
}

Published online: 15 November 2016

(C) Springer International Publishing AG 2016

Women sleep differently than men due to physiological, psychological, and social reasons. From estrogen receptor modulation of sleep in the brain, to an increased risk of experiencing the correlated disorders of depression and insomnia, to the impact of poverty and relationship satisfaction on sleep - both biological sex and the construct of female gender affect the timing, quantity, and quality of women's sleep. It is now known that women sleep longer and report that they need more sleep than men. Furthermore, despite showing higher quality sleep than men in most objective sleep studies, women tend to report more sleep disturbances than men. Although not all of the reasons for these observations are known or understood, we are beginning to make some progress.

As one of us shared in The Woman's Book of Sleep: A Complete Resource Guide [1], women's health became a national priority with the founding of the NIH Office of Research on Women's Health in the USA in 1990 and the CDC Office of Women's Health in 1994. Of course, women make up about $50 \%$ of the world population and yet historically the health needs of women have lagged in medical and health care research. Over the last 25 years, there has been a transformation in women's health research, including changes in government and private support of research, in policies, and in organization that has generated new knowledge about women's health. Since that time and particularly in the last

Katherine M. Sharkey

katherine_sharkey@brown.edu

Amy R. Wolfson

awolfson@loyola.edu

1 Alpert Medical School of Brown University, Providence, RI, USA

2 Loyola University Maryland, Baltimore, MD, USA decade, research and clinical practice devoted to women's sleep patterns and disorders have grown significantly as well.

The articles in this special issue of Current Sleep Medicine Reports address some of the recent progress made in multiple facets of women's sleep. In their paper, Sleep, Circadian Rhythms, and Fertility, Drs. Goldstein and Smith review the evidence that sleep and circadian rhythms impact successful reproduction through central and peripheral mechanisms and propose that sleep and circadian dysregulation may be a modifiable risk factor for couples experiencing infertility. Dr. O'Brien's contribution, Sleep Disordered Breathing in Pregnancy, provides a state-of-the-art update on how the continuum of sleep-disordered breathing - from snoring to acute "gestational" sleep apnea to "chronic" co-incident sleep apnea-can negatively impact the health of both mother and infant. Turning to sleep disorders, Drs. Krystal and Attarian's paper, Sleep Medications and Women: a Review of Issues to Consider for Optimizing the Care of Women with Sleep Disorders, discusses evidence that the agents zolpidem and modafinil/armodafinil, commonly used in insomnia and hypersomnia, respectively, have different pharmacologic effects in women than in men and provide practical tips for clinicians who utilize these medications in their practice. Finally, Drs. Byun, Lerdal, Gay, and Lee's article, How Adult Caregiving Impacts Sleep: A Systematic Review, summarizes the current knowledge about how a woman's role as caregiver for a demented, critically ill or chronically ill family member impacts her sleep. Taken together, the papers in this Topical Collection touch on how sleep is related to many aspects of women's lives across the lifespan.

Certainly women are not a homogenous group and it is important to consider how stage in the life influences women's sleep. Women's sleep is clearly a complex and multifaceted phenomenon. The papers in this volume exemplify the progress that has been made in understanding the unique interplay 
between sleep and circadian rhythms and women's health. Looking ahead and with the third NIH research agenda for women's health, Moving into the Future with New Dimensions and Strategies for Women's Health Research: A Vision for 2020 for Women's Health Research [2], sleep researchers will have the opportunity to continue to advance the understanding of gender, gender role, and sex differences in sleep patterns and disorders and translate basic sleep research into new interventions across health care fields. It is our hope that these papers will inspire further work that advances our understanding of the role of sleep and circadian rhythm health in women's well-being.

\section{Compliance With Ethical Standards}

Conflict of interest Katherine M. Sharkey and Amy R. Wolfson declare that they have no conflict of interest.

Human and Animal Rights and Informed Consent This article does not contain any studies with human or animal subjects performed by any of the authors.

\section{References}

1. Wolfson AR, Lee KA. The woman's book of sleep: a complete resource guide. Oakland: New Harbinger; 2001.

2. A vision for 2020 for women's health research: moving into the future with new dimensions and strategies. NIH Publication No. 10-7606. 